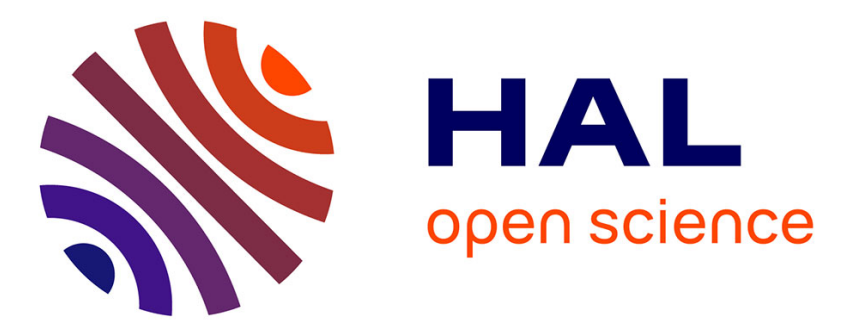

\title{
Co-design for wireless networked control of an intelligent mobile robot
}

Amine Mechraoui, Zeashan Hameed Khan, Jean-Marc Thiriet, Sylviane Gentil

\section{To cite this version:}

Amine Mechraoui, Zeashan Hameed Khan, Jean-Marc Thiriet, Sylviane Gentil. Co-design for wireless networked control of an intelligent mobile robot. ICINCO 2009 - 6th International Conference on Informatics in Control, Automation and Robotics, Jul 2009, Milan, Italy. pp.7. hal-00396510

\section{HAL Id: hal-00396510 https://hal.science/hal-00396510}

Submitted on 18 Jun 2009

HAL is a multi-disciplinary open access archive for the deposit and dissemination of scientific research documents, whether they are published or not. The documents may come from teaching and research institutions in France or abroad, or from public or private research centers.
L'archive ouverte pluridisciplinaire HAL, est destinée au dépôt et à la diffusion de documents scientifiques de niveau recherche, publiés ou non, émanant des établissements d'enseignement et de recherche français ou étrangers, des laboratoires publics ou privés. 


\title{
Co-design for wireless networked control of an intelligent mobile robot
}

\author{
Amine MECHRAOUI, Zeashan Hameed KHAN, Jean-Marc THIRIET, Sylviane GENTIL \\ Gipsa-Lab, Control departement INPG-UJF-CNRS, BP 46, 38402 Saint Martin d'Hères \\ amine.mechraoui, zeashan-hameed.khan, jean-marc.thiriet, sylviane.gentil@gipsa-lab.inpg.fr
}

\begin{abstract}
Keywords: Autonomous mobile robots, Networked control systems, Wireless network, 802.15.4 protocol, Horizontal handoff, TrueTime simulation.

Abstract: $\quad$ This paper describes a wireless network based control of a Khepera mobile robot moving in a distributed infrastructure. Due to critical dependence on wireless communication, a procedure for reconfiguration of the network is proposed as a possibility to maintain communication between control station and the mobile robot in a successful manner. The network handoff is made under a criterion that takes into account key application dependent performance parameters. The controlled system and the communication network are simulated respectively with Matlab/Simulink and TrueTime.
\end{abstract}

\section{INTRODUCTION}

Networked control systems (NCS) in mobile robotics are getting very popular today. With the rapid progress in communication techniques, especially the wireless networks, distributed control and decision have become mandatory to reduce the onboard processing overhead. It includes control, decision, obstacle avoidance etc; as it effects the battery consumption in miniature robots with space and weight as key design constraints. However, introducing a wireless network in the control loops presents some disadvantages such as band limited channels, sampling delays and packet dropouts (Hespanha et al., 2007). Furthermore, the mobility of the robot also adds some problems, e.g. increasing the distance between the control station and the robot increases the number of lost packets due to decreased signal strength and increased bit error rate (BER) (Zhu et al., 2005).

The communication architecture in mobile robotics may be centralized, in which case there is a fixed or mobile node, which communicates with all the other nodes, or decentralized, where individual mobile nodes should ideally operate without any central control (Schwager et al., 2007). In the decentralized control scheme, each component solves a part of the problem and shares memory without having a global view of the mission. There is less emphasis on computation than communication. In distributed control systems, communication is an important parameter and individual components don't need to share memory (Martinez et al., 2007). In related research work, many approaches have been used for distributed control of mobile robots. In (Fierro and Lewis, 1996) the dynamic model of the mobile robot is controlled by means of neural networks. In (Aicardi et al., 1995) and (canudas de Wit and Sordalen, 1992), a nonlinear control approach has been introduced. Another research area, related to the hybrid architecture of control for autonomous navigation robots is studied in (Benzerrouk et al., 2008).

The objective of this paper is to define a communication architecture for a mobile robot moving in a 2D space with several fixed stations (wireless infrastructure communication). According to the position of the robot, communication is possible in a specific coverage area with one or several stations (see Fig. 1). When several stations are reachable, the robot will choose one of the stations (Horizontal Handoff (HHO) strategy (Wang et al., 2007)) that can allocate sufficient resources to ensure a good level of communication. This comprises of optimal choice of payload and delay based on distance between robot and station and thus maintaining the necessary Quality of Service (QoS) in order to ensure that the Quality of Control (QoC) is sufficient (distributed control). When there is no station in the reachable space, the robot will have to be absolutely autonomous (embedded control), maintaining a sufficient QoC despite a degradation of the communication QoS. The QoC in wireless NCS is defined as the performance delivered by each closed-loop operation. Stability is the main property that must be guaranteed but control error and response time are also important to analyse. 


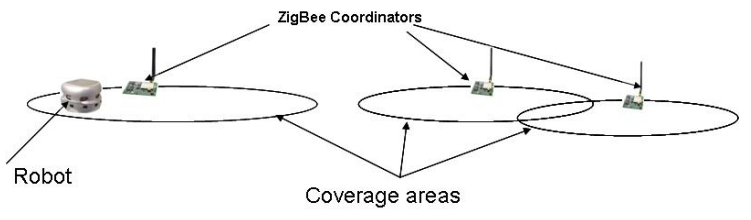

Figure 1: Problem description

In the literature, many researchers have proposed a HHO strategy. They proposed making a decision by taking into account the Received Signal Strength (RSS), the power consumption and the cost of communication (Chen et al., 2004). We propose to add the packet dropouts caused by the propagation delay or the distance and orientation between the robot and the station, which have a consequence on the QoC.

The paper is organized as follows. The second section presents a brief description of the model of the Khepera robot, notably the kinematic model and the dynamic one. In the third section, the controller design is described and simulations of the tracking trajectory are presented. Section 4 presents the control over ZigBee wireless network and the influence of the integration of this network on control performance. After that, the proposed HHO architecture is described with one, two and three stations. Finally, the conclusion and perspectives are presented.

\section{MODELING AND CONTROL}

This section presents the study of a unicycle Khepera robot (Lambercy and Caprari, 2007). Consider a unicycle robot (Khepera) as shown in Fig. 2. Let $\mathrm{x}, \mathrm{y}$ and $\theta$ be the state variables where $x \in \mathfrak{R}$ and $y \in \Re$ are the Cartesian coordinates, $\theta \in[0,2 \pi[$ is the robot's orientation with respect to the X-axis. We consider ' $v$ ' and ' $\omega$ ' respectively as the linear and the angular velocities of the robot. The kinematic of the robot can be modeled as

$$
\dot{x}=v \cos \theta, \quad \dot{y}=v \sin \theta, \quad \dot{\theta}=\omega
$$

The kinematics model of the mobile robot has two control inputs $\omega_{\text {left }}$ and $\omega_{\text {right }}$ i.e. the left and right wheels velocities. These are related to the linear velocity $v$ and the angular velocity $\omega$ of the robot according to the following equations

$$
V_{\text {right }}=v+R \omega, \quad V_{\text {left }}=v-R \omega
$$

where $R$ is half the distance between the two robot's wheels. The dynamic model of the robot wheels is characterized by the equations of the DC motors driving the wheels. They are represented by a first order model

$$
\frac{\omega_{(\text {left }, \text { right })}^{*}}{U}=\frac{K}{\tau s+1}
$$

where $U$ is the voltage applied to the motor and $\omega_{(\text {left,right })}^{*}$ are angular velocities generated by each motor. $\tau$ is the time constant $(\tau=0.63 s)$ and $K$ is the gain $(K=5.3)$.

\subsection{Controller Design}

The objective of this section is to present how to control the robot to track any trajectory. Two levels of controllers are required. The first one is needed to control the angular velocities of the motors. PI controllers are implemented. The second one controls the linear and angular velocities of the robot. Let us consider the controller presented in (Toibero et al., 2007), where the robot can reach a desired target point $\left[\begin{array}{lll}x_{d} & y_{d} & \theta_{d}\end{array}\right]$. Errors are defined as

$$
\tilde{x}=x_{d}-x, \tilde{y}=y_{d}-y
$$

and the tracking error (Eq. 5a) and the orientation error (Eq. 5b) are calculated as

$$
\begin{gathered}
d=\sqrt{\widetilde{x}^{2}+\widetilde{y}^{2}} \\
\widetilde{\theta}=\theta_{d}-\theta=\tan ^{-1}(\widetilde{y} / \widetilde{x})-\theta
\end{gathered}
$$

According to (Toibero et al., 2007), the following control actions are defined

$$
\begin{gathered}
v=\frac{v_{\max }}{1+|d|} d \cos (\widetilde{\theta}) \\
\omega=\frac{v_{\max }}{1+|d|} \cos (\widetilde{\boldsymbol{\theta}}) \sin (\widetilde{\boldsymbol{\theta}})+K_{\widetilde{\theta}} \tanh \left(k_{\theta} \widetilde{\theta}\right)
\end{gathered}
$$

where $v_{\max }$ is the maximum linear velocity that the robot can reach $\left(v_{\max }=0.3 \mathrm{~m} / \mathrm{s}\right)$ and $K_{\widetilde{\theta}}, k_{\theta}$ are constants. Those controllers are stable according to (Toibero et al., 2007) using the Lyapunov candidate function $V_{t}$.

$$
V_{t}=\widetilde{\theta}^{2} / 2+d^{2} / 2
$$

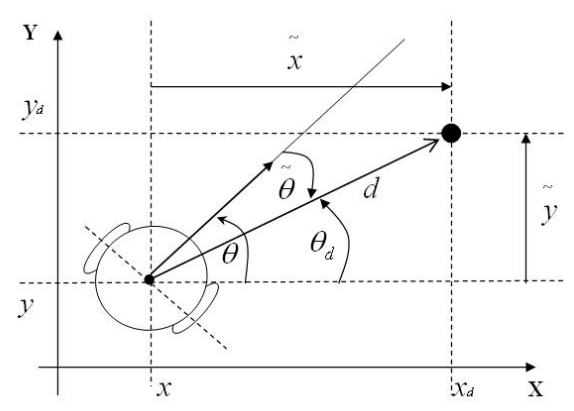

Figure 2: Robot Model 


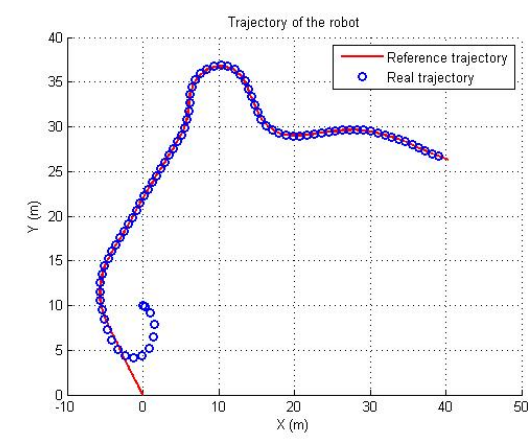

Figure 3: Real trajectory of the robot

Fig. 3 shows the reference trajectory with initial conditions $\left[X_{0}, Y_{0}, \theta_{0}\right]=[0,10,0]$ and the real trajectory of the robot using those controllers. $X, Y$ and $\theta$ are measured each sampling time $\left(T_{s}=0.03 \mathrm{~s}\right)$. These results are obtained with Matlab/TrueTime. The PI controllers are discretized and the voltage applied to the motor is obtained with a zero order hold. The trajectory is known in advance and the references $x_{d}, y_{d}$ and $\theta_{d}$ are updated every $0.28 \mathrm{~s}$.

\section{CONTROL OVER NETWORK}

In this section, the controller is digitized and Zigbee wireless network is integrated (see Fig. 4). The effects of network on control system are analyzed.

\subsection{WPAN 802.15.4}

ZigBee is a specification for small, low-power digital radios based on the IEEE 802.15.4 standard for wireless personal area networks (WPANs). The low cost allows the technology to be widely deployed in wireless control and monitoring applications. The low power-usage allows longer life with smaller batteries. The mesh networking provides high reliability and larger range. The main reasons for adopting this wireless network in our application is its low power

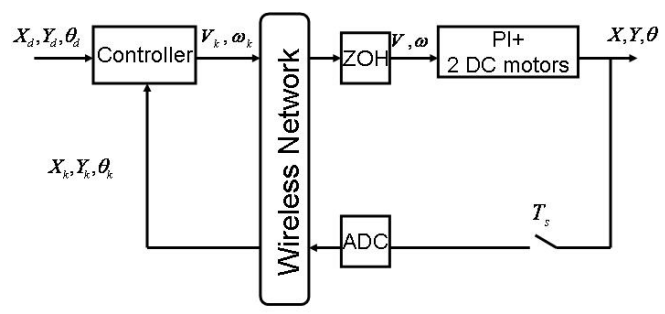

Figure 4: Control over Zigbee network consumption and its ad-hoc networking capabilities.

\subsection{Control over Zigbee}

Control feedback loops are closed through a real-time network as shown in Fig. 4.

To perform this study, the following conditions are considered:

- A bit rate of $250 \mathrm{~kb} / \mathrm{s}$ for Zigbee in the physical layer.

- The sensor flow and the controller flow use 248 bits each. The sensor flow uses $3.3 \%$ and the controller one uses $3.3 \%$ of the network capacity with a sampling period $T_{s}$ equal to $30 \mathrm{~ms}$.

TrueTime simulator is used to simulate Zigbee Network. Two tasks are programmed, the first is the controller task that generates the controller flow and the second one is a periodic sensor task that generates sensors flow. The controller task is event-triggered, which means that the controller calculates and sends the control signals $V_{k}$ and $\omega_{k}$ when it has received all measures $X_{k}, Y_{k}$ and $\theta_{k}$. With the TrueTime Simulation of ZigBee Network, there is only the CAP period (Contention Access Period which uses CSMA/CA protocol), therefore there is no priority mode like CFP (Contention Free Period) which allows Guaranteed Time Slots (GTS) (see (van den Bossche et al., 2007) for more information about 802.15.4 protocol).

The sensor and control data are critical to keep the stability of the system, hence packet losses are undesirable. Practically, loss of packets cannot be entirely eliminated in wireless networks. A lot of methods e.g. Forward Error Correction (FEC) (Kurose and Ross, 2004) are proposed to reduce the probability of packet losses. The retransmission of lost packets is proposed too but for a real-time application, this is an inappropriate solution. When a packet has been discovered to be lost and is retransmitted, the sensors state evolve to a newer one and thus the retransmitted packet will be based on the old information and the calculated control will be wrong. The solution to ensure the best control is to do over sampling, with a sampling rate higher than what is needed, which therefore makes control more tolerant to packet losses. However, this solution increases the Use Request Factor (URF) and causes more packet delays and losses (Mechraoui et al., 2008). The minimal sampling period to ensure a good QoC is $0.3 s$ in our example. To increase the QoC over wireless network and decrease the probability of losing packets of critical data, the sampling period of $30 \mathrm{~ms}$ is required. Fig. 5 shows that the robot can communicate with the station only within 


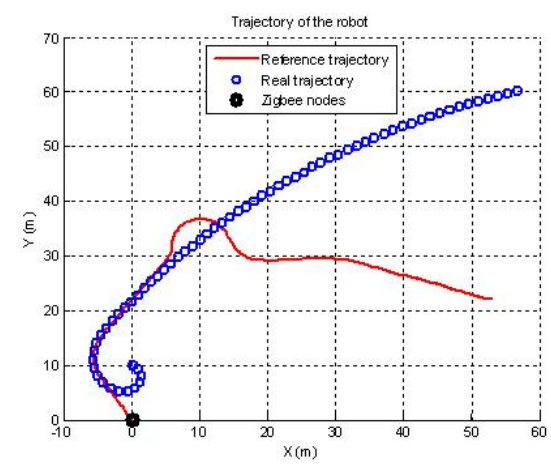

Figure 5: Trajectory of the robot with one Zigbee station

the coverage range of the station. When it losses the communication, it keeps the last value of the linear and angular velocities and it deviates from the reference trajectory. The progress of WPAN and the utilisation of this kind of wireless networks allow the robot to find another station (PAN) to communicate for control. Therefore, we assume that we have different stations that the robot can communicate with. The next section explains how to change stations to ensure sufficient QoS.

\section{STATION SELECTION}

\subsection{Handoff Decision}

For several stations, mobility management is proposed through redundancy in network communication by searching for the best available protocol based on a cost function. Horizontal and vertical handoff can be proposed. A horizontal handoff (HHO) is the link transfer between two network access points that use the same network technology while vertical handoff (VHO) is between two network access points using different network technologies. (Chen et al., 2004) describes a smart decision model to decide the best network interface and best time for VHO. (StevensNavarro and Wong, 2006) gives a comparison between four different VHO decision algorithms w.r.t to bandwidth and delay for different traffic classes. (Wang et al., 1999) describes a policy-enabled handoff system that allows users to express policies, based on the estimation of current network conditions and determining the best network at any moment by characteristics e.g. cost, performance and power consumption. (Nkansa-Gyekye and Agbinya, 2007) proposes a distributed additive weighting based VHO mechanism to reduce the processing overhead in the

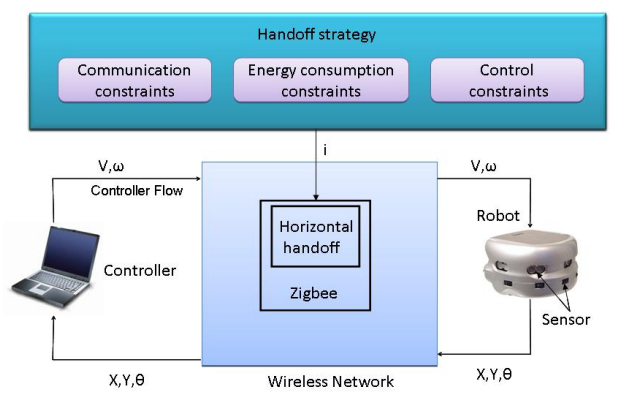

Figure 6: Network HHO strategy for a mobile robot control

mobile terminal by delegating the calculation of handoff metrics for network selection to the visiting networks. (Angermann and Kammann, 2002) evaluates different structures for cost functions and presents a simulation for the influence of various parameters. The challenge in handoff is to maintain the application session alive while the physical connection interface is changed. A generic weighted 'Network Rating Function' evaluation for each network is based on

$$
\begin{gathered}
N R F_{i}=W_{R S S I} N_{R S S I, i}+W_{N L} N_{N L, i}+W_{D} N_{D, i} \\
W_{R S S I}+W_{N L}+W_{D}=1
\end{gathered}
$$

where $W_{R S S I}, W_{N L}$ and $W_{D}$ are weighting functions of RSSI, network load (NL) and delay (D) respectively. $N_{R S S I, i}, N_{N L, i}$ and $N_{D, i}$ indicate the scores of interface. $N R F_{i}$ is between 0 and $1 . W_{i}$ is the weight of the factor $i$, which emphasizes the importance of each contributing factor and $N_{i, j}$ represents the normalized score of the interface ${ }^{\prime} j$ for factor ${ }^{\prime} i$.

The embedded logic in the mobile robot, assigns different "weights" to the handoff decision parameters in order to determine the level of importance of each parameter for the information to be exchanged between neighbors. In our case $W_{R S S I}>W_{N L}>W_{D}$. The best station connection interface at any given moment is then chosen as the one that achieves the highest score among all candidate interfaces.

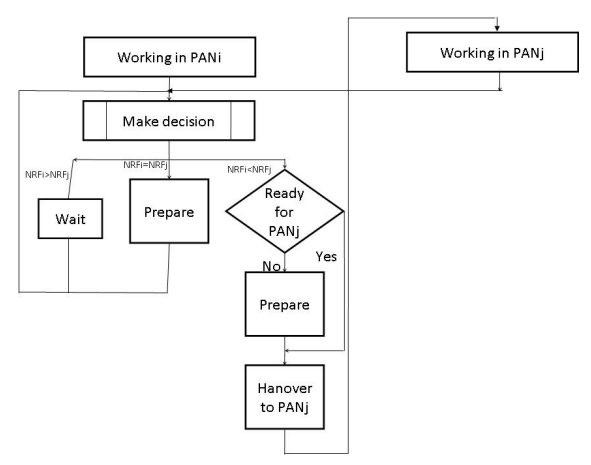

Figure 7: HHO state diagram 
In our scenario, factors within $N_{i, j}$ include QoS parameters i.e. the RSSI, the network load $(N L)$ and Delay or latency $(D)$ of the candidate network. Now we calculate $N R F_{i}$ and $N R F_{j}$ to choose accordingly (which ever is greater) the station that maximizes the decision criteria (Fig. 7).

Additionally, there is a corresponding function for each term $N_{R S S I, i}, N_{N L, i}$, and $N_{D, i}$, and the ranges of the functions are bounded between 0 and 1 . The functions are given in Eq. (10).

$$
\begin{gathered}
N_{R S S I, i}=\frac{e^{P_{r}}}{e^{P_{s}}} \\
N_{N L, i}=1 / e^{N L} \mid 0 \leq N L \leq 1 \\
N_{D, i}= \begin{cases}1 & \text { if } S_{i} \text { is the current station \& } R S S I>-48 \mathrm{dBm} \\
0 & \text { if not }\end{cases}
\end{gathered}
$$

In our case, the transmitted power is equal to $-3 \mathrm{dBm}$ and the receiver signal threshold is equal to $-48 \mathrm{dBm}$.

The RSSI is calculed as follow

$$
\begin{aligned}
P_{r} & =\frac{1}{d^{\alpha}} P_{S} \\
R S S I(d B m) & =10 \log \left(P_{r}(m W)\right)
\end{aligned}
$$

where $P_{r}$ and $P_{s}$ are the power in $m W$ received and sent respectively. $d$ is the distance between the two nodes in meters, and $\alpha$ is a parameter that can be chosen to model different environments.

Fig. 8 shows the trajectory of the robot with changes in station if necessary (QoS is the best to ensure the best QoC). The stations positions are

- For the first station $\left(S_{1}\right)(0,5)(\mathrm{m})$

- For the second station $\left(S_{2}\right)(-3,30)$

- For the last station $\left(S_{3}\right)(40,35)$.

For this experiment, the weight values are $W_{R S S I}=$ $0.8, W_{N L}=0.15$ and $W_{D}=0.05$.

We assume that $S_{1}$ and $S_{2}$ have a common coverage area, and $S_{3}$ is far. According to the initial position of the robot (here $\left.\left(X_{0}, Y_{0}, \theta_{0}\right)=(0,0,0)\right)$ the robot communicates first with $S 1$. It continues its trajectory until the robot detects the second station. In this case, Khepera executes Algorithm 1.

To understand this algorithm, symbols are used. $(\mathrm{R})$ is the mobile robot, $S_{i}$ is the current station, $S_{\{1,2, \ldots k\}}$ are all the stations and $S_{\{m, \ldots, n\}}$ are the stations that are detected by the robot. We note the fact that the robot detects a set of stations by the symbol $\bowtie$.

When the robot is out of range of all stations, it keeps the last values of the angular and the linear velocities which is not a good solution because it can

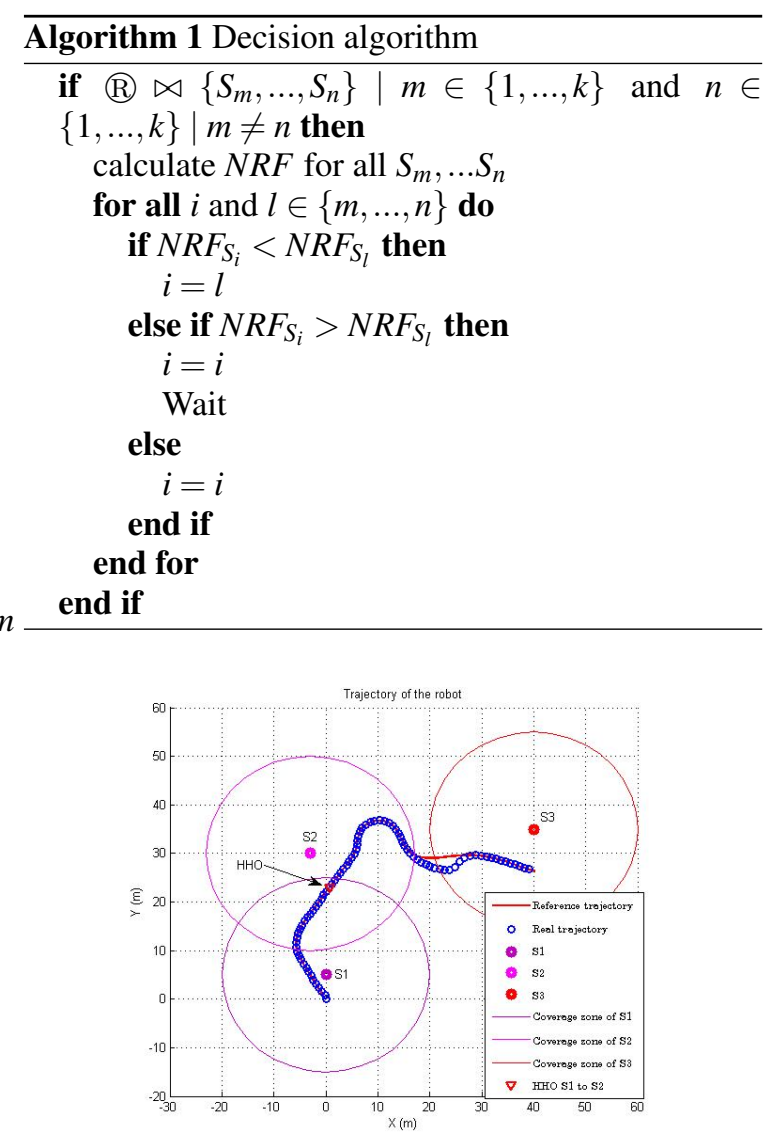

Figure 8: Trajectory of the robot with $\mathrm{HHO}$ with $\mathrm{NL}=0$
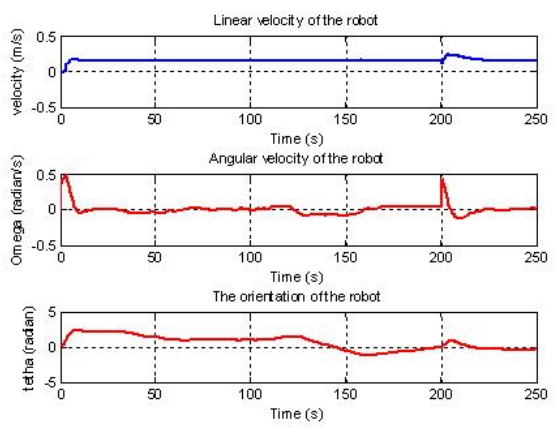

Figure 9: Evolution of the linear and angular velocities

result in an important error with respect to the reference trajectory and cause problems (see Fig.8). We propose in this case to change the controller and use an embedded one.

Figures 10 and 11 show the change of stations. In Fig.10, the simulation is made with $\mathrm{NL}=0$ for all stations. The change from $S_{1}$ to $S_{2}$ depends thus on the distance between the robot and the station. The robot 


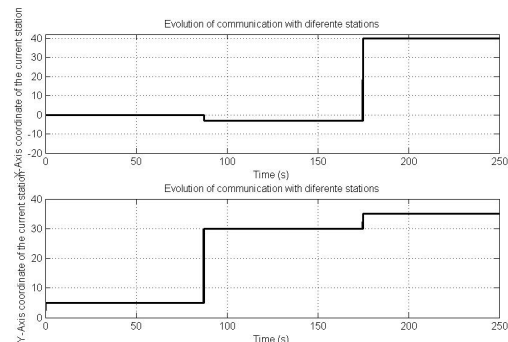

Figure 10: Evolution of communication with different stations with $\mathrm{HHO}$ with $\mathrm{NL}=0$ (corresponds to Fig.8)

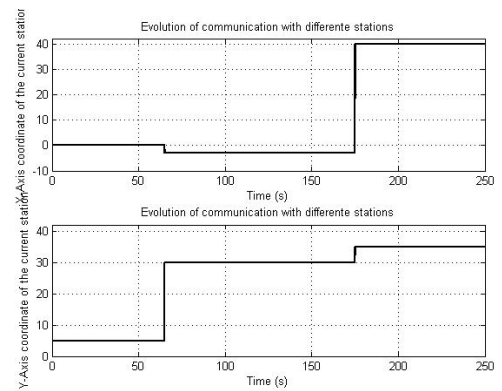

Figure 11: Evolution of communication with different stations with $\mathrm{HHO}$ with $N L_{S_{1}}=0.8$ and $N L_{S_{2}}=0.2$

changes the station only if the RSSI of the current station is poor. In Fig.11, the simulation is made under the assumption that the $N L_{S_{1}}=0.8$ which means that the network is $80 \%$ loaded and $N L_{S_{2}}=0.2$. In this scenario the robot decides to change the station according to the RSSI of both stations and the network load of each station. Comparing Fig. 10 and Fig. 11 the $\mathrm{HHO}$ of the station is quite sensitive to parameter $N L$.

\subsection{Control Decision}

As the robot moves outward towards another control station, the signal strength decreases. The low value of RSSI is sensed by the mobile robot, which then requests the station for an estimation of next way-point. The control station sends the trajectory information as well as broadcasts the "standby" message to all control stations in the infrastructure to minimize the time of connection of robot for the next cell. Thus, a PAN slot is reserved in advance for the coming robot as the trajectory is already estimated. This proposition permits to maintain a sufficient QoC despite a degradation of the QoS.

The embedded control design is the same than the distributed one. The only difference is that the sampling period is changed. The controller computes control signals each $0.3 \mathrm{~s}$. This change is very important to reduce the energy consumption and also

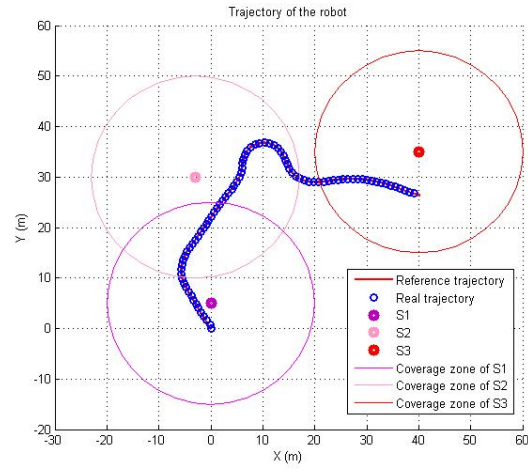

Figure 12: The trajectory of the robot with switching controller
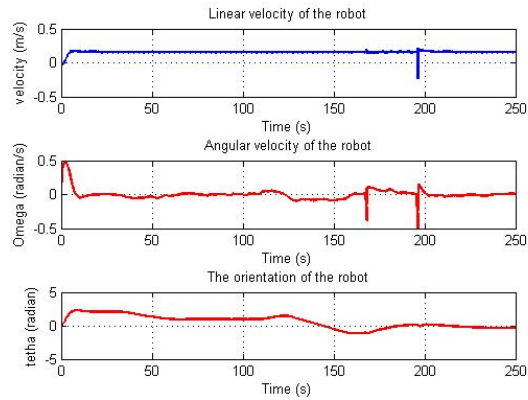

Figure 13: Evolution of linear and angular velocities of the robot with switching controller

the execution time. Algorithm 1 and 2 is executed ("poor" in Algorithm 2 means that the RSSI of the current station comes near the receiver signal threshold). Fig.12 show the simulation when the control is switched within the non covered area.

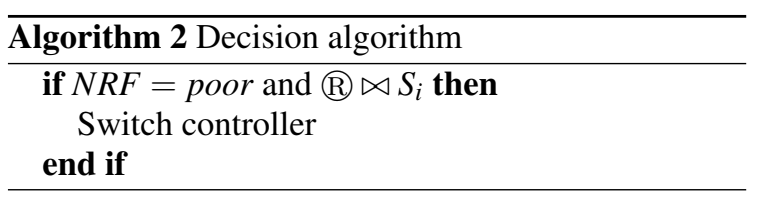

\section{CONCLUSION}

This work aims to study, in a co-design approach, the influence of a wireless network QoS on the QoC of a teleoperated robot. A wireless network based control of a unicycle mobile robot in a distributed infrastructure mode is described. The simulations are performed using Matlab/TrueTime toolbox. The problem faced was a loss of communication when the mobile 
robot moves out of range or there is an excessive network load that prohibits successful communication. The first strategy consists of adapting the network QoS to the control requirements. An algorithm for reconfiguration by $\mathrm{HHO}$ is proposed as a solution to maintain communication between control stations and the mobile robot in multi stations scenarios. The $\mathrm{HHO}$ is made with a criterion that takes into account the QoC for the robot as well as the QoS of wireless network.

The second strategy deals with the reconfiguration of the control, for the robot to be autonomous, when the communication link is out of order. Therefore, if the robot is out of range of all stations, the control mode is switched to embedded control, increasing the sampling period to reduce computations and the robot is completely autonomous. Integration of WLAN could be a choice for extended zone coverage for mobile robots.

In the future work, a quantification of QoS and QoC will be dealt. A combination of infrastructure and adhoc architecture will also be investigated in order to maintain sufficient QoC in multi robots perspective.

\section{Acknowledgements}

This work is partially supported by the Safe NeCS project funded by the French Agence Nationale de la Recherche under grant ANR-05-SSIA-0015-03.

\section{REFERENCES}

Aicardi, M., Casalino, G., Bicchi, A., and Balestrino, A. (1995). Closed loop steering of unicycle like vehicles via Lyapunov techniques. In IEEE Robotics and $\mathrm{Au}$ tomation Magazine, volume 2(1), pages 27-35.

Angermann, M. and Kammann, J. (2002). Cost metrics for decision problems in wireless ad-hoc networking. In IEEE CAS Workshop on Wireless Communications and Networking, Pasadena, California, USA.

Benzerrouk, A., Adouane, L., Martinet, P., and Andreff, N. (2008). Toward an hybrid control architecture for a mobile multi-robot systems. In Proceeding of the $3^{\text {rd }}$ National Conference on Control Architectures of Robots, Bourges, France.

canudas de Wit, C. and Sordalen, O. (1992). Exponential stabilization of mobile robots with non-holonomic constraints. In IEEE Trans. Automatic Control, volume 37, pages 1792-1797.

Chen, L.-J., Sun, T., Chen, B., Rajendran, V., and Gerla, M. (2004). A smart decision model for vertical handoff. In Proceedings of the $4^{\text {th }}$ International Workshop on Wireless Internet and Reconfigurability, Athens, Greece.

Fierro, R. and Lewis, F. (1996). Practical point stabilization of a nonholonomic mobile robot using neural net- works. In Proceeding Conference on Decision and Control, Kobel, Japan.

Hespanha, J., Naghshtabrizi, P., and Xu, Y. (2007). A survey of recent results in networked control systems. In Proceeding of IEEE special issue on Technology of Networked Control Systems, volume 95, pages 138162.

Kurose, J. and Ross, K. W. (2004). Computer Networking: A Top-Down Approach Featuring the internet. Addison Wesley, $3^{r d}$ edition.

Lambercy, F. and Caprari, G. (2007). Khepera3 user manual version 2.2. Technical report.

Martinez, S., Corts, J., and Bullo, F. (2007). Motion coordination with distributed information. In IEEE Control Systems Magazine, volume 27(4), pages 75-88.

Mechraoui, A., Thiriet, J.-M., and Gentil, S. (2008). Control of an intelligent robot system over a wireless network. In Proceeding of the $23^{\text {rd }}$ IAR Workshop on Advanced Control and Diagnosis, Coventry, United Kingdom.

Nkansa-Gyekye, Y. and Agbinya, J. (2007). Vertical handoff decision algorithm for umts-wlan. In Proceeding of the $2^{\text {nd }}$ International Conference on Wireless Broadband and Ultra Wideband Communications, Sydney, Australia.

Schwager, M., Slotine, J., and Rus, D. (2007). Decentralized, adaptive control for coverage with networked robots. In Proceeding of IEEE International Conference on Robotics and Automation, Roma, Italy.

Stevens-Navarro, E. and Wong, V. (2006). Comparison between vertical handoff decision algorithms for heterogeneous wireless networks. In Proceeding of the $63^{\text {rd }}$ IEEE Vehicular Technology Conference, volume 2, pages 947-951.

Toibero, J. M., Carelli, R., and Kuchen, B. (2007). Switching control of mobile robots for autonomous navigation in unknown environments. In Proceeding of IEEE International Conference on Robotics and Automation, Roma, Italy.

van den Bossche, A., Val, T., and Campo, E. (2007). Prototyping and performance analysis of a qos mac layer for industrial wireless network. In Proceeding of the $7^{\text {th }}$ IFAC International Conference on Fieldbuses and Networks in Industrial and Embedded Systems, Toulouse, France.

Wang, H., Katz, R., and Giese, J. (1999). Policy-enabled handoffs across heterogeneous wireless networks. In Proceeding of the $2^{\text {nd }}$ IEEE Workshop on Mobile Computing Systems and Applications, New Orleans, Louisiana, USA.

Wang, L., Chen, A., and Chen, H. (2007). Network selection with joint vertical and horizontal handoff in heterogeneous wlan and mobile wimax systems. In Proceedings of the $63^{\text {rd }}$ IEEE Vehicular Technology Conference, Melbourne, Australia.

Zhu, H., Yang, Q., and Kwak, K. (2005). Performance analysis of fast handoff with mobility prediction. In Proceeding of IEEE International Symposium on Communications and Information Technology, Beijing, China. 